\title{
Patrimonio histórico versus patrimonio moderno. Problemática de conservación del Edificio de los Poderes de Campeche, México
}

Historical Heritage versus Modern Heritage. Conservation Issues Concerning the Edificio de los Poderes (Building of Powers) in Campeche, Mexico.

\author{
Raúl González Medina \\ Arquitecto independiente, México \\ glez_raul@hotmail.com

\begin{abstract}
Aurelio Sánchez Suárez
Centro de Investigaciones Regionales (CIR),

Universidad Autónoma de Yucatán (UADY), México

aurelio_suarez@yahoo.com.mx
\end{abstract}

\section{Resumen}

La subvaloración y un inconcluso proceso de apropiación son factores que han propiciado la destrucción del patrimonio moderno. Campeche, México, es ejemplo, como otras ciudades con patrimonio moderno, de esta paulatina destrucción patrimonial. Con edificaciones representativas de la arquitectura moderna, la ciudad amurallada de Campeche convivía con las diferentes categorías de patrimonio construido en su centro histórico, hasta la llegada de la propuesta de un Campeche esencialmente colonial, que aceleró la destrucción del patrimonio moderno. En este trabajo nos enfocamos en el caso del Edificio de los Poderes por ser el más representativo; no obstante, existe patrimonio moderno que está siendo afectado y es de vital importancia pensar en su protección.

\section{Palabras clave}

patrimonio moderno; patrimonio histórico; conservación; Campeche; México

\section{Abstract}

Modern heritage has been destroyed because of its undervalued status and because of an inconclusive process of appropriation. Campeche, Mexico, is an example of a place that, like 
other cities with modern heritage, has suffered the gradual demolition of its cultural heritage. With the arrival of the proposal of conceiving Campeche as an essentially colonial city, the destruction of modern heritage accelerated: until then buildings representing modern architecture cohabited with constructions of other periods in the historical centre of the walled city. This CHRONICLE focuses on the case of the Edificio de los Poderes (Building of Powers) as the most representative example of modern heritage that is being affected. There are, however, other modern heritage buildings under threat that are in urgent need for protection.

\section{Key words}

modern heritage; historical heritage; conservation; Campeche; Mexico

\section{Introducción}

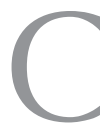

on el pasar de los siglos se han expuesto muchas ideas y criterios sobre la protección del patrimonio $^{1}$ y se ha creado una conciencia respecto de ella, lo que denota tanto un enriquecimiento progresivo como, implícitamente, la creación de un lenguaje más fino y específico para su denominación, clasificación y preservación. La colaboración de múltiples organizaciones y autores, así como las nuevas investigaciones en materia de cultura, han contribuido a dicha evolución.

El tema de la conservación del patrimonio siempre ha sido controversial, en especial cuando se trata de la valoración de qué patrimonio es más importante o tiene más valor de conservación que otro (Muñoz Viñas 2003). La misma Ley Federal sobre Monumentos y Zonas Arqueológicos, Artísticos e Históricos (CEUM 1972) en México establece categorías de patrimonio que dan preponderancia al arqueológico, protegen muy claramente al histórico, pero dejan en la ambigüedad y, por lo tanto, indefenso, al moderno, circunscrito al rubro "monumentos artísticos". Esto ha propiciado que, durante decenios, muchas ciudades, en su acelerado proceso de novedad y crecimiento, hayan sufrido pérdidas en lo que se refiere al patrimonio arquitectónico del siglo Xx (Calduch Cervera 2009).

En México la protección del patrimonio arquitectónico moderno se encuentra en problemas debido a diversos factores, entre ellos, la falta de actualización de instrumentos legales para su protección, el desconocimiento y

\footnotetext{
${ }^{1}$ Hablar de los criterios y conceptos es hablar de la historia y la teoría de la restauración, tema extenso que no es el objeto del trabajo; sin embargo, es importante recalcar que siempre ha existido pluralidad en las ideas. Muchos teóricos han generado aportaciones al campo de la restauración desde sus experiencias y estudios, destacando en México a Salvador Díaz-Berrio y Carlos Chanfón Olmos, que crearon corrientes teóricas. Componentes también de la esfera teórica son las cartas internacionales, que van generando nuevos conceptos y definiendo categorías.
}

la falta de difusión de sus valores, y la pérdida de archivos relacionados.

En lo que se refiere a la ciudad de San Francisco de Campeche, la producción de arquitectura moderna fue escasa; de sus edificios destacan los construidos durante el gobierno del entonces coronel José Ortiz Ávila (19611967), a cargo del arquitecto Joaquín Álvarez Ordóñez. En este periodo la ciudad sufrió alteraciones en su estructura y la pérdida de significativos monumentos históricos ante la construcción de edificios con un nuevo lenguaje formal, alejado de historicismos y con propuestas espaciales novedosas, además de fuentes, monumentos conmemorativos y avenidas, como el Ilamado Edificio de los Poderes, la Cámara de Diputados, el Centro Cívico Héctor Pérez Martínez, la Plaza de la República, el mercado Pedro Sainz de Baranda y las fuentes de las Estelas, de los Pescadores y de la Nacionalización (GEC 1963).

Campeche, una vez más, está cambiando rápidamente, y así como durante la década de 1960 se perdieron importantes edificios coloniales, en la actualidad las intervenciones, enfocadas en la reconstrucción de la ciudad amurallada, que busca resaltar el patrimonio histórico, ponen en peligro las construcciones realizadas durante el siglo $\mathrm{xx}$, lo que refleja la poca valoración de una arquitectura a la que en 1994 se señaló como "inserciones de construcciones modernas y discordantes con el contexto histórico, como es el caso del palacio de gobierno y la Cámara de Diputados de la ciudad de Campeche" (INAH 2000:110). En esta tendencia, el patrimonio moderno: la arquitectura doméstica, la educativa, los edificios públicos y el arte urbano, se ha alterado o destruido. En esta contribución describiremos la valoración del patrimonio moderno y las afectaciones de un solo ejemplo de los muchos que hay en la ciudad, esto es, del Edificio de los Poderes.

\section{La ciudad de Campeche y José Ortiz Ávila}

El desarrollo de la ciudad de Campeche ha estado condicionado desde su fundación, en 1540, por su ubicación frente al mar. La villa crecería con base en el modelo renacentista de traza rectangular, con una plaza excéntrica concebida como el centro religioso, político y comercial de la población (Lanz 1905; Leal Sosa 2003), y, por ser la única salida para muchos productos de interés para Europa y Estados Unidos, como el tabaco, la sal y el palo de Campeche, su actividad marítima y comercial la convertiría en el centro económico más importante de la península de Yucatán durante los siglos XVII y XVIII (Lanz 1905). Ya en la segunda mitad del siglo XIX, con la creación de otros puertos en la zona, Campeche dejó de ser un punto clave, por lo que disminuyó su actividad económica. Entonces, aislada del resto del país por tierra y con un puerto marítimo en decadencia, la ciudad entró en un periodo de poco desarrollo durante el siglo XIX y la primera mitad del siglo Xx (Lanz 1905; Pérez Sosa 2000). 
Con la construcción del Ferrocarril del Sureste y del Circuito del Golfo y del Caribe, a mediados del siglo pasado, Campeche logró integrarse nuevamente con el resto del territorio nacional: el gobierno federal consideró en dicho momento que la ciudad representaba un punto estratégico para la integración física y socioeconómica de la península, pues, además de ser un punto de paso obligado dentro de dicho circuito, era puerto pesquero y de cabotaje (GEC 1963).

En 1961 comenzó un nuevo periodo gubernamental en la entidad, el del licenciado y coronel José Ortiz Ávila, que se caracterizaría por la promoción de grandes beneficios para el estado: se realizaron más de 3000 obras, entre las que sobresalen la pavimentación de carreteras y la construcción de numerosos edificios públicos, escuelas y centros de salud (GEC 1963). También destacó la colonización del sur del estado, que hizo posible la llegada de más de 10000 personas sin tierras que, provenientes de otros estados, formaron nuevas colonias que con los años han prosperado (GEC 1963). Ortiz Ávila fomentó, de igual manera, la industria apícola y pesquera, convirtiendo la miel campechana en un producto de exportación y duplicando la captura de especies marinas, con una flota pesquera que aumentó de 256 a 492 embarcaciones (GEC 1963; Sierra 1998).

Asimismo, durante el sexenio de Ortiz Ávila, la nueva condición territorial del estado dio como resultado la creación de un nuevo programa de desarrollo urbano para el futuro crecimiento de la ciudad capital, el cual se proponía la redistribución de la población y la ampliación del casco urbano mediante la reorganización lógica y uniforme de los usos y vialidades para satisfacer de manera adecuada las necesidades de sus habitantes (cfr. GEC 1963). El programa estaba basado parcialmente en el plan de desarrollo elaborado por el arquitecto y urbanista Domingo García Ramos durante el sexenio del licenciado Manuel López Hernández (1949-1955): el Campeche Nuevo - que ejecutaría su sucesor, el doctor Alberto Trueba Urbina (GEC 1963)—, consistente en ganar terrenos al mar, en los que se construirían nuevos edificios y vialidades (GEC 1963).

A pesar de las intenciones de conservar intacto el Campeche intramuros, las transformaciones realizadas durante 1961 y 1962 con base en el nuevo plan regulador tuvieron como consecuencia la pérdida de edificios del periodo virreinal e independiente, sobre todo en el primer cuadro de la ciudad, donde se demolieron el mercado, la aduana, los palacios de gobierno y municipal, así como la arquería morisca del edificio Cuauhtémoc, en cuyo sitio se erigieron los portales Revolución. Todas estas edificaciones formaron parte del patrimonio cultural material de Campeche que ahora podemos conocer sólo por fotografías y por la memoria de los ancianos habitantes de la ciudad. ${ }^{2}$

\footnotetext{
${ }^{2}$ La arquería, constituida por arcos mixtilíneos, era de las pocas en
}

\section{El Edificio de los Poderes}

El 20 de junio de 1962, el arquitecto Joaquín Álvarez Ordóñez dirigió una carta al coronel Ortiz Ávila, con copia para el señor arquitecto Guillermo Rossell de la Lama, subsecretario del Patrimonio Nacional, en la que exponía un diagnóstico del antiguo palacio de gobierno para que se decidiera acerca de su demolición y la edificación de uno nuevo:

Antiguo palacio de gobierno:

Aspecto urbanístico. El palacio de gobierno presentaba serios problemas de localización urbanística [...]: Invadía la vía pública y taponaba en forma de embudo la circulación de la Calle 8. Tapaba el viejo y bellísimo lienzo de la muralla a la que estaba adosado arbitrariamente. Ahogaba la proporción y perspectiva de la plaza.

Aspecto arquitectónico. Tenía nulo valor arquitectónico en virtud de que no conservaba ningún estilo determinado y constituía un hacinamiento desordenado de construcciones hechas en el transcurso de los 100 últimos años.

Aspecto ingenieril. Debido a lo viejo de la construcción, ésta se encontraba en muy malas condiciones de estabilidad, ya que las vigas de madera estaban todas podridas, constituyendo un peligro constante de derrumbe, ya que en festividades y recepciones había que distribuir a la gente con el fin de repartir la carga en toda el área, sintiéndose claramente la vibración y el movimiento de los pisos al caminar sobre ellos. Los techos y los muros se encontraban fuertemente dañados y en condiciones muy deficientes.

Aspecto funcional. El edificio no satisfacía el área ni las condiciones de funcionamiento necesarias en este tipo de edificaciones. La gran cantidad de área desperdiciada en patios y corredores hacía difícil y cara su conservación (GEC 1962).

El mismo documento hacía mención respecto del futuro destino del terreno del antiguo palacio después de su demolición:

a) Deberá de eliminarse el tapón que constituía el palacio de gobierno en la calle para lo que deberá alinearse la calle al Eje que viene desde el Mercado.

b) Se aumentará el área de la Plaza hasta el alineamiento que viene de la acera poniente de la misma calle.

c) Se construirán las banquetas correspondientes en

\footnotetext{
América que estaban construidas en una plaza mayor, por lo que su pérdida es invaluable. El edificio del palacio de gobierno, que se erigió haciendo caso omiso de la muralla y del paseo de ronda frente al mar, constaba, hasta marzo de 1962, de tres secciones: gobierno del estado, H. Ayuntamiento y aduana. La noticia más lejana de este edificio informa que se encontraba funcionando con dos pisos: el palacio municipal, al centro; a la derecha, un piso que servía de juzgado de distrito, y a la izquierda una arquería de cuatro arcos, de un piso también, que se Ilamaba El Principal (Lanz 1905).
} 
ambos lados de la calle, teniendo la del lado de la muralla un ancho de 1.80 [metros].

d) En el espacio sobrante entre la baqueta y el muro se sembrará pasto logrando un motivo decorativo en ese espacio a base de una fuente o un espejo de agua con una combinación de plataformas de diferentes pavimentos y a diferente nivel, donde se localicen figuras o estelas originales y de valor arqueológico.

e) La muralla se regenerará limpiándola de todo material extraño y se rejoneará con piedra en aquellos lugares donde se encuentre lastimada.

f) A la muralla se le aplicará el procedimiento necesario para "patinarla" a fin de darle el aspecto que presentan todos los demás tramos de murallas.

g) Para lograr el mejor funcionamiento de la muralla ésta se iluminará con luz de reflectores empotrados en muretes colocados sobre el césped.

h) El espacio que está situado en el interior del fuerte se ajardinará, iluminándose los muros en la forma ya descrita.

i) Se cerrará el espacio exterior con una reja que será de la misma forma de la existente en el Museo.

j) Se respetará rigurosa y absolutamente la muralla y todo aquello que tenga valor arquitectónico o histórico (GEC 1962).

De lo anterior resaltamos el poco interés que se tenía en el antiguo palacio de gobierno, al cual, pese a que formaba parte de los edificios representativos de la plaza mayor, se lo consideró de limitado valor — la prioridad era el lienzo amurallado-y se le acusó de generar una alteración de la morfología original del puerto, con su Plaza de Armas excéntrica dando al mar, lo que resultó en la demolición del inmueble.

La triste pérdida de este y otros edificios que formaron parte de la identidad del campechano dio paso a una nueva generación, que conformó el patrimonio moderno de la ciudad de Campeche que hoy está enfrentando problemas no sólo para su conservación sino para su existencia.

\section{Unidad de gobierno y Plaza de la República}

El Edificio de los Poderes, como se describió anteriormente, es un diseño que integra dos elementos: la unidad de gobierno y la Plaza de la República, ambos construidos en la ciudad y puerto de Campeche a inicios del sexenio de José Ortiz Ávila, durante el periodo comprendido entre septiembre de 1961 y diciembre de 1962 (Figuras 1 y 2). Más tarde, en enero de 1963, lo inauguraría el presidente Adolfo López Mateos, como se había anunciado desde el principio del proyecto. La obra fue propuesta a solicitud del Gobierno del Estado de Campeche (GEC), y diseñada por el arquitecto Joaquín Álvarez Ordóñez, en colaboración con alumnos de la Universidad Nacional Autónoma de México (UNAM) de la generación 1959 (GEC 1963).
En relación con la Plaza de la República, el 29 de agosto de 1962 se estaba realizando el colado de las losas que formarían a sus costados el "serpenteado de cantera", lo que conceptualizaba el espíritu nacionalista de ese periodo.

De una carta escrita por el arquitecto Álvarez Ordóñez, dirigida al coronel Ortiz Ávila, con fecha 20 de junio de 1962, se obtuvieron los siguientes datos sobre el diseño del Edificio de los Poderes:

Aspecto funcional:

a) El espacio resultante (mismo que ocupara el antiguo palacio de gobierno) después de alinear la calle y la plaza es sumamente reducido para localizar adecuadamente todas las dependencias que deben estar incluidas dentro del edificio y sería imposible levantar una construcción en dicho lugar porque taparía la muralla que es de extraordinaria belleza y que forma parte del patrimonio histórico de la ciudad.

b) Si el edificio se localiza partiendo sobre columnas a fin de permitir la vista de la muralla a través del mismo, entonces habría necesidad de levantar una construcción de dos o más pisos, lo cual no sería de ninguna forma conveniente, pues desproporcionaría las dimensiones horizontales de la plaza, en la cual deben destacarse como valor vertical las torres de la Catedral, permaneciendo las edificaciones de la plaza en proporciones acentuadamente horizontales.

c) Sería oportuno aprovechar este momento para regenerar el estilo arquitectónico de la Plaza Principal logrando una armónica proporción de sus formas y colores, suprimiendo anuncios y elementos que no presentan valores de consideración arquitectónica o histórica.

Aspecto arquitectónico:

a) No habría de pensarse en construir un edificio moderno en el lugar donde se encontraba el Palacio antiguo, pues su arquitectura resultaría impropia y demasiado contrastada con el ambiente general del lugar.

b) De ninguna manera habría que sustituirlo, igualmente, por un edificio de estilo antiguo, pues aparte de ser un engaño estético, esto haría desmerecer el valor arquitectónico de los edificios que sí lo tienen verdaderamente ya que se trata de resaltar lo auténtico no confundiéndolo con falsos valores y anacrónicas imitaciones; resultaría inútil, pero debe apuntarse, lo doloroso que sería hacer un edificio en esas condiciones.

c) Es por ello que es absolutamente necesario respetar en esencia la traza de la vieja ciudad no construyendo edificios modernos ni tampoco edificios de falso estilo. Esta observancia vendría a aumentar el valor histórico del recinto amurallado, caso en el cual el Gobierno daría ejemplo de respeto y cultura al preservarse de violarlo.

Por lo anterior se deduce lo siguiente:

a) Construir un edificio moderno con las condiciones ne- 


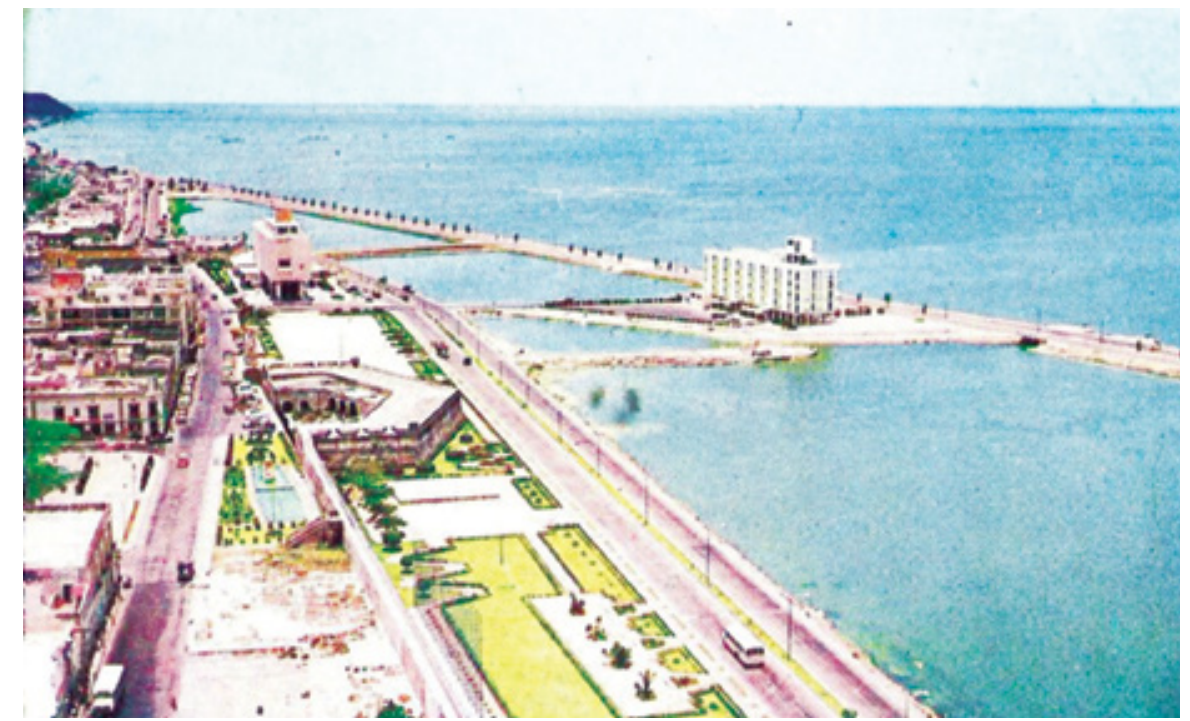

FIGURA 1. Vista aérea de la Unidad de gobierno, ciudad de Campeche, Campeche, México, en su diseño original (Fuente: Postal Mex Photocolor s. f.; cortesía: colección González Medina).

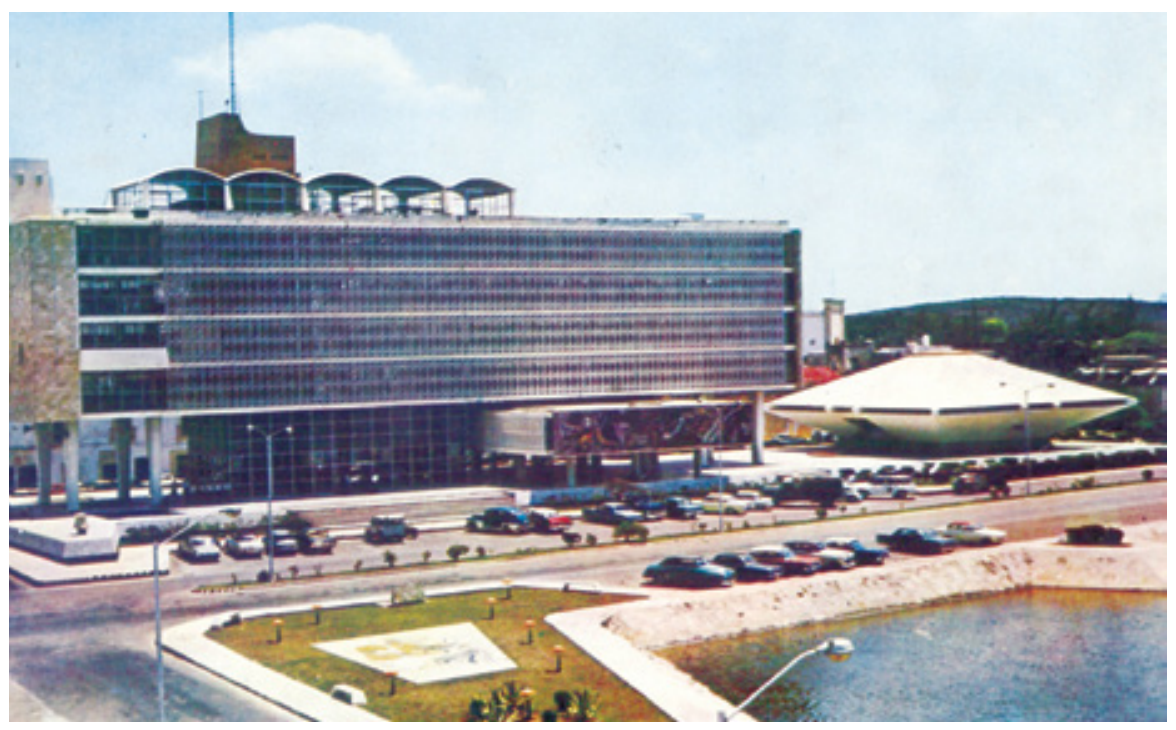

FIGURA 2. Vista de la fachada norte del Edificio de los Poderes, ciudad de Campeche, Campeche, México, con su diseño original (Fuente: Postal Mex Photocolor s. f.; cortesía: colección González Médina).

cesarias para el buen funcionamiento de las oficinas gubernamentales, fuera del recinto amurallado y en la zona céntrica más próxima a la Plaza de Armas, ubicándose de preferencia en un lugar que procure dar el espacio y la perspectiva en relación con la importancia y dignidad del Edificio de los Poderes.

No corresponde a ningún ordenamiento urbanístico el ubicar forzosamente el Edificio de los Poderes del Estado en la Plaza de Armas. De esto existen innumerables ejemplos en otras Capitales de Estados.

b) Habrá que tender a ir generando en forma inductiva para lograr el valor e importancia a la zona donde se ubique el nuevo edificio. c) Desde el punto de vista arquitectónico hay que considerar que el edificio en cuestión deberá tener las condiciones de dignidad propias de los edificios de Gobierno.

d) A la vez deberá estar concebido en relación con los recursos económicos con que cuenta el Estado y de acuerdo con la tónica revolucionaria del Gobierno de Campeche. Será oportuno el cambio de nombre que desea darle el C. Gobernador al nuevo edificio al designarlo con el nombre de "Edificio de los Poderes", en vez del inadecuado y tradicional "Palacio de Gobierno". Arq. Joaquín Álvarez Ordóñez (GEC 1962).

El análisis, la disertación y las propuestas del arquitecto Álvarez Ordóñez no discordaban con lo que se estaba cimentando en el pensamiento de la conservación del patrimonio arquitectónico - recuérdese que los planteó en tiempos en que la teoría de la restauración estaba a punto de concretar la "Carta de Venecia" (UNESCO 1964)—: si bien no respetaba la historicidad del antiguo palacio de gobierno, el cual pudo haber sido restaurado y darle otro uso, sí mostraba interés por preservar el espacio urbano, el paisaje y las escalas originales de la Plaza de Armas; abogaba, asimismo, por la construcción de un edificio que evitara los falsos valores y anacrónicas imitaciones del estilo arquitectónico del periodo colonial. Respetaba la traza del recinto amurallado en una propuesta encaminada a una nueva arquitectura que no sólo fuera diferente estéticamente sino también en su significado, por lo que la construcción recibió el nombre de Edificio de los Poderes, eliminando el acostumbrado título de palacio, aunque éste no pudo ser borrado de la memoria colectiva.

De los tres posibles sitios para la ubicación del futuro Edificio de los Poderes que propuso el arquitecto Álvarez Ordóñez: el situado frente al Cuartel Federal, entre la Calle 8 y la Avenida 16 de Septiembre; segundo, construir en el espacio de agua señalado, que quedaba entre las avenidas 16 de Septiembre, Ruiz Cortines, y la Calle 61, y, tercero, levantarlo entre las avenidas 16 de Septiembre y Ruiz Cortines, en el espacio de agua ubicado en la parte más importante del llamado Campeche Nuevo; se escogió, por razones que se desconocen, el primero. 
Este predio, de $3381 \mathrm{~m}^{2}$, forma parte de los terrenos ganados al mar durante el sexenio del doctor Alberto Trueba Urbina (1955-1961), cuyo propietario era el profesor Rafael Alcalá Dondé, quien lo compró al gobierno del estado el 27 de julio de 1961 por 60000 pesos, misma cantidad que recibió tras donarlo de manera irrevocable al propietario original (GEC 1962). A éste se sumó el predio que se encuentra al otro lado de la Calle 61, entre la Calle 8 y la Avenida 16 de Septiembre, donde anteriormente estuvo el muelle de la ciudad, que data de 1777, era la continuación de la Calle 59 y tenía aproximadamente $16 \mathrm{~m}$ de ancho y $125 \mathrm{~m}$ de largo (GEC 1962). Tras la realización del proyecto del Campeche Nuevo, este terreno, que era propiedad de la federación, fue donado al Ayuntamiento de la ciudad, con fecha de 30 de diciembre de 1957, por decreto presidencial (GEC 1962).

La Plaza de la República y la unidad de gobierno, destinados a formar el nuevo centro cívico de la ciudad de Campeche, donde, aparte de estar emplazados los poderes del estado, se realizarían los actos de orden cívico y cultural que en épocas anteriores se realizaban en la Plaza de la Independencia, constituían el punto central y más importante de los nuevos espacios que se construían a la par alrededor del recinto amurallado en el nuevo Circuito Baluartes y en los terrenos ganados al mar. En la Plaza de la República se encuentra la escultura de un águila estilizada que simboliza a la patria, proyectada con motivos regionales de carácter moderno (GEC 1962; GEC 1963).

La unidad de gobierno se edificó sobre un basamento de $1.20 \mathrm{~m}$ de altura, en el que se encuentra el estacionamiento, y está compuesto por dos cuerpos principales. El primero de ellos, que era el mezzanine, contenía las oficinas de mayor concurrencia pública, cuyas fachadas longitudinales cuentan con murales con tema de la Revolución realizados por el muralista mexicano José Chávez Morado, que son un bello ejemplo de la integración, buscada en esos tiempos, entre el arte y la arquitectura (GEC 1963). El segundo - y mayor- cuerpo, que contenía las oficinas de los poderes Ejecutivo y Judicial, así como la presidencia municipal, se resolvió en cuatro niveles, en cuyas fachadas longitudinales se aprecia una celosía fabricada de manera expresa para el edificio. Particularmente en la que encara a la Plaza de la República se encuentra un balcón y, a su lado, el escudo de la ciudad de Campeche, tallado por el maestro Arturo Gil Mendicuti por encargo del entonces gobernador Manuel López Hernández para el antiguo palacio de gobierno, el cual se rescató durante su demolición (GEC 1963). Este cuerpo tiene como remate una campana, igualmente rescatada del antiguo palacio, que se utilizó en la ceremonia del 15 de septiembre, y en su azotea, un helipuerto y un salón de usos múltiples a los cuales se tenía acceso directo desde la oficina del gobernador (GEC 1962; GEC 1963).

Parte del patrimonio inmueble por destino del edificio son los dos murales de mosaico, de $3 \mathrm{~m}$ por $18 \mathrm{~m}$ cada uno, fabricados con piedras de diferentes colores, ubicados en el área del mezzanine. El mural del sur, Historia de Campeche, da a la Calle 8 del centro histórico, encarando a la ciudad antigua, a manera de homenaje a sus antiguos constructores mayas y españoles, y en su última parte, al lado derecho, vemos a un hombre de rasgos mestizos que, ubicado a un costado del nuevo edificio, apunta hacia la lejanía mostrándonos el camino hacia nuevos tiempos. Por otro lado, en el mural norte, Los constructores de Campeche, que se encuentra del lado del Campeche Nuevo, vemos al indígena que libera sus cadenas para dar paso a la construcción, precisamente, de un nuevo Campeche (GEC 1963; Silva 2001). Estos mosaicos están en excelentes condiciones, pues a principios de 2009 se restauraron, con lo que recobraron los vivos colores que se habían perdido con el paso del tiempo.

Anexo en la parte posterior, sobre el mismo basamento que el palacio de gobierno, se encuentra el edificio de forma octaédrica, originalmente rodeado por un espejo de agua, que alberga a la Cámara de Diputados y se utiliza para la celebración tanto de las sesiones del Congreso del Estado como de otros actos cívicos. En el basamento de este edificio se localizan las oficinas del Congreso, dispuestas alrededor de un patio central en un esquema en forma de $U$, a las que se accede descendiendo por unas escalinatas que se encuentran en la Calle 8 y en la Avenida 16 de Septiembre y que comunican a una pequeña plaza a nivel del sótano, entre el basamento del conjunto y el baluarte de San Carlos.

Frente a la fachada frontal del Edificio de los Poderes, al otro lado de la Calle 61, se ubica la Plaza de la República, compuesta por tres secciones. La central es una plaza dura destinada a actos de tipo cívico y cultural. En dicho espacio, dentro de una fuente, se encuentra la escultura de un águila - a la que ya nos hemos referido-, la cual, junto con dos astas, forma el remate visual de la plaza. Los cuerpos laterales están constituidos por áreas verdes delimitadas por medio de un murete recubierto de cantera que, con diseño de greca, recorre la plaza de manera longitudinal, representando el cuerpo de la serpiente (GEC 1962; GEC 1963).

Este conjunto, a pesar de estar próximo a la villa antigua y de haberse proyectado con un lenguaje moderno, propio de sus tiempos, permitía que la ciudad siguiera teniendo la vista al mar (que recuperó cuando fue destruida la muralla del tramo en el que se ubica la puerta de tierra), ya que los edificios de gobierno se encontraban desplantados sobre pilotes, influencia — podría considerarse- de los "cinco puntos de una nueva arquitectura" de Le Corbusier; es decir, esto permitía conservar la imagen de ciudad puerto desde el recinto amurallado. De igual manera, la construcción de la Plaza de la República consintió la permanecía del espacio en el que anteriormente se encontraba el muelle fiscal y permite observar sin mayor obstáculo tanto los restos del sistema defensivo como los edificios históricos situados a lo largo de la 
Calle 8, resultado - asumimos- de las intenciones de conservación expresadas por el arquitecto Álvarez Ordóñez en sus cartas al coronel Ortiz Ávila.

Con el paso de los años, el conjunto ha sufrido cambios que han operado no sólo en su detrimento sino también en el de su entorno, en su mayoría, como consecuencia de que aquél, extralimitado en su capacidad, se ha visto alterado por ampliaciones que no han considerado la esencia formal del edificio.

Las primeras modificaciones que sufrió el edificio — de cuya data no encontramos información- fueron la eliminación del espejo de agua que rodeaba el volumen de la Cámara de Diputados y la ampliación del pequeño cubo de cristal que albergaba originalmente las circulaciones verticales. En la actualidad este cuerpo de cristal cubre la mayor parte del basamento y cuenta con un entrepiso en la parte frontal del edificio que, además de impedir la vista hacia el mar desde la Calle 8, entre la 61 y la 63 , también afecta la naturaleza formal del inmueble.

Durante el periodo gubernamental 1985-1991 se agregó el elevador que comunica directamente la oficina del gobernador con el estacionamiento subterráneo. Al siguiente periodo (1991-1997) se eliminó el remate del volumen principal que comprendía el helipuerto y el salón de usos múltiples. Durante 19972003 se retiró la escalera que encaraba a la Plaza de la República y se colocaron macetas de gran tamaño en el basamento del edificio, ocasionando la ruptura de la circulación directa a la plaza y el primero de los daños al concepto del conjunto, quedando sólo el balcón gubernamental y la campana como única vinculación entre la plaza y el Edificio de los Poderes (Figura 3).

La Plaza de la República (Figuras 4,5 y 6) no queda exenta de modificaciones. Dentro del espíritu de la inscripción en la Lista de Patrimonio Mundial de la ciudad amurallada de Campeche, el gobierno del estado

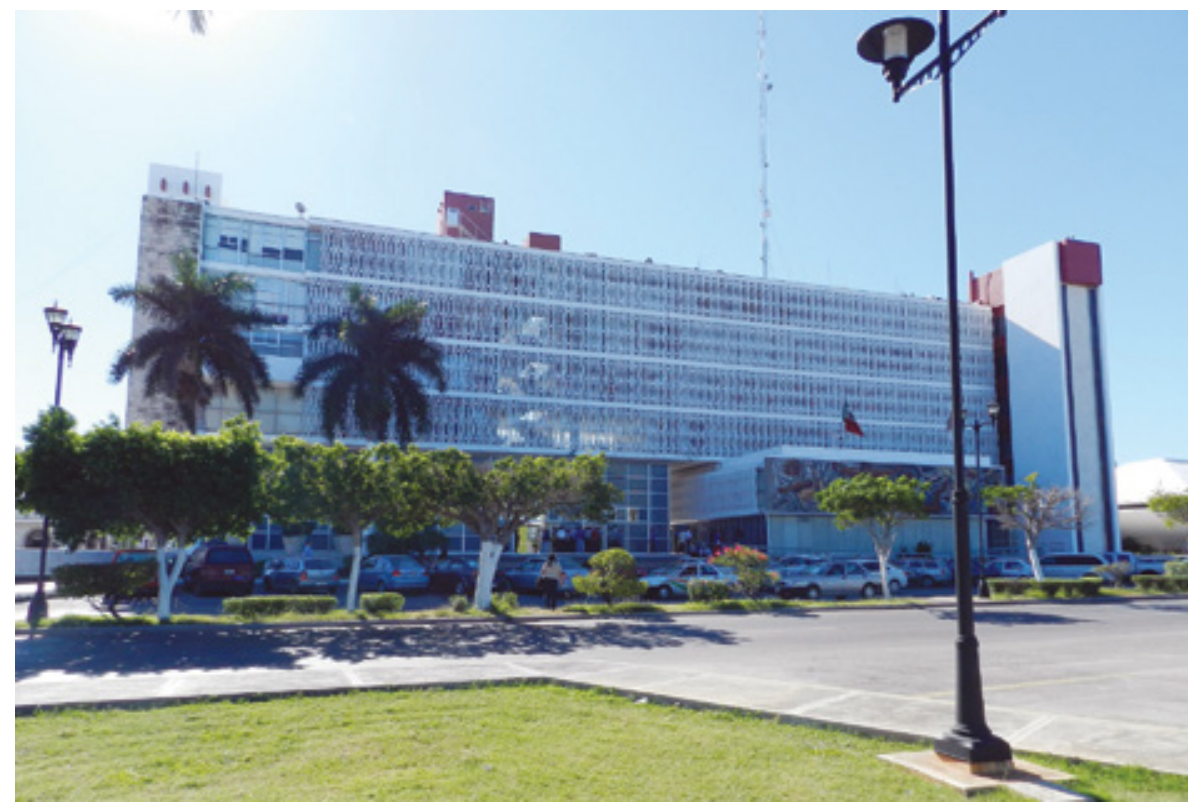

FIGURA 3. Vista de la fachada norte del Edificio de los Poderes, ciudad de Campeche, Campeche, México, con el volumen agregado para la construcción del elevador (Fotografía: Raúl González Medina 2013).

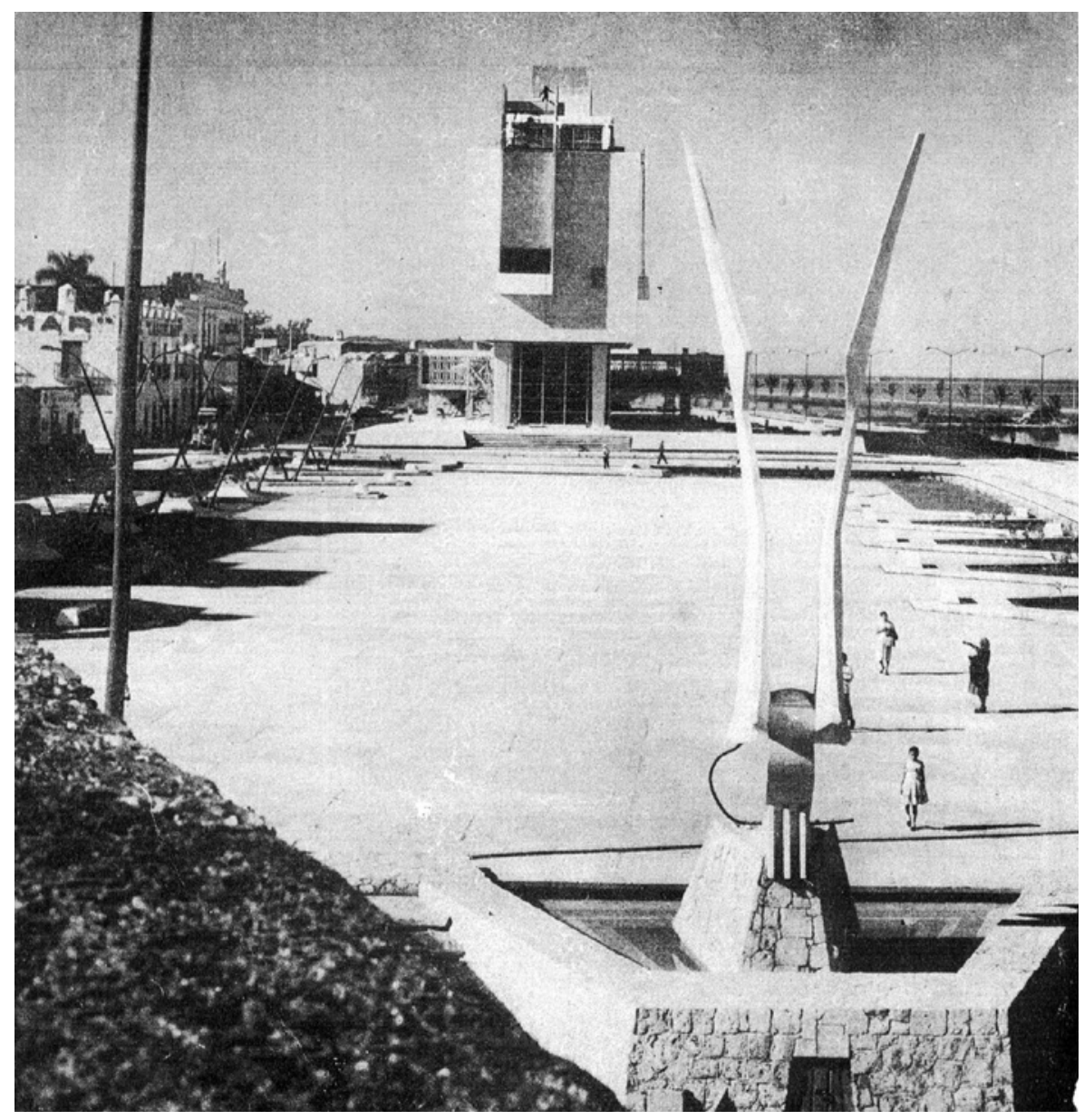

FIGURA 4. Imagen de la Plaza de la República, ciudad de Campeche, Campeche, México, en la que se observan las escalinatas que vinculan al Edificio de los Poderes con la plaza, vista desde el Baluarte de la Soledad (Fuente: GEC 1963). 


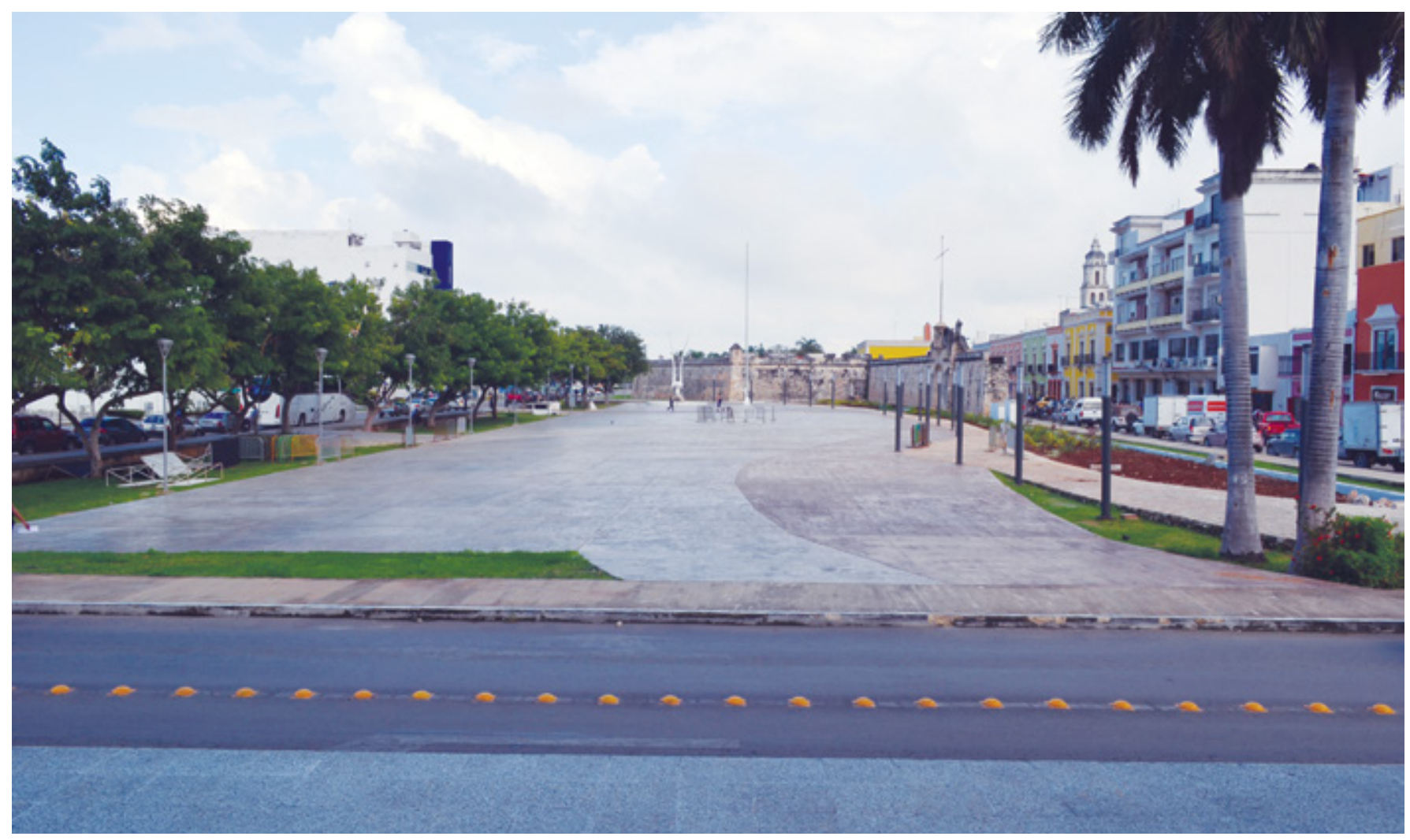

FIGURA 5. Imagen de la Plaza de la República desde el Edificio de los Poderes, ciudad de Campeche, Campeche, México, en la actualidad, con la remodelación realizada (Fotografía: Aurelio Sánchez 2013).

comenzó una serie de acciones para resaltar el Campeche colonial, por lo que se propuso la reconstrucción de la muralla en el tramo donde se ubica la puerta de mar. De la propuesta presentada, ${ }^{3}$ sólo se reconstruyó el tramo que unió la puerta de mar con el baluarte de la Soledad; el paisaje arquitectónico no se vio afectado, pero con el incremento vehicular y la falta de estacionamientos públicos, el gobierno (2003-2009) tomó la decisión de utilizar la plaza como estacionamiento, como se pudo apreciar a lo largo de varios años en la ciudad de Campeche. Durante los años 2013 y 2014 se siguieron reconstruyendo lienzos de muralla de la parte sur y oriente de lo que era el recinto amurallado.

\section{Reflexiones finales}

En la actualidad podemos apreciar los cambios que se están dando en la capital del estado de Campeche, principalmente con la reconstrucción de la muralla. Las intervenciones realizadas en la ciudad intramuros y en los barrios tradicionales, fruto del desconocimiento, la mala

\footnotetext{
${ }^{3}$ El ICOMOS Yucatán solicitó al Centro INAH-Campeche y a la Secretaría de Cultura de la entidad información sobre el proyecto; sólo se obtuvo la contenida en la presentación dada a los medios de comunicación, pero no se contó con la relativa a un proyecto de restauración o conservación.
}

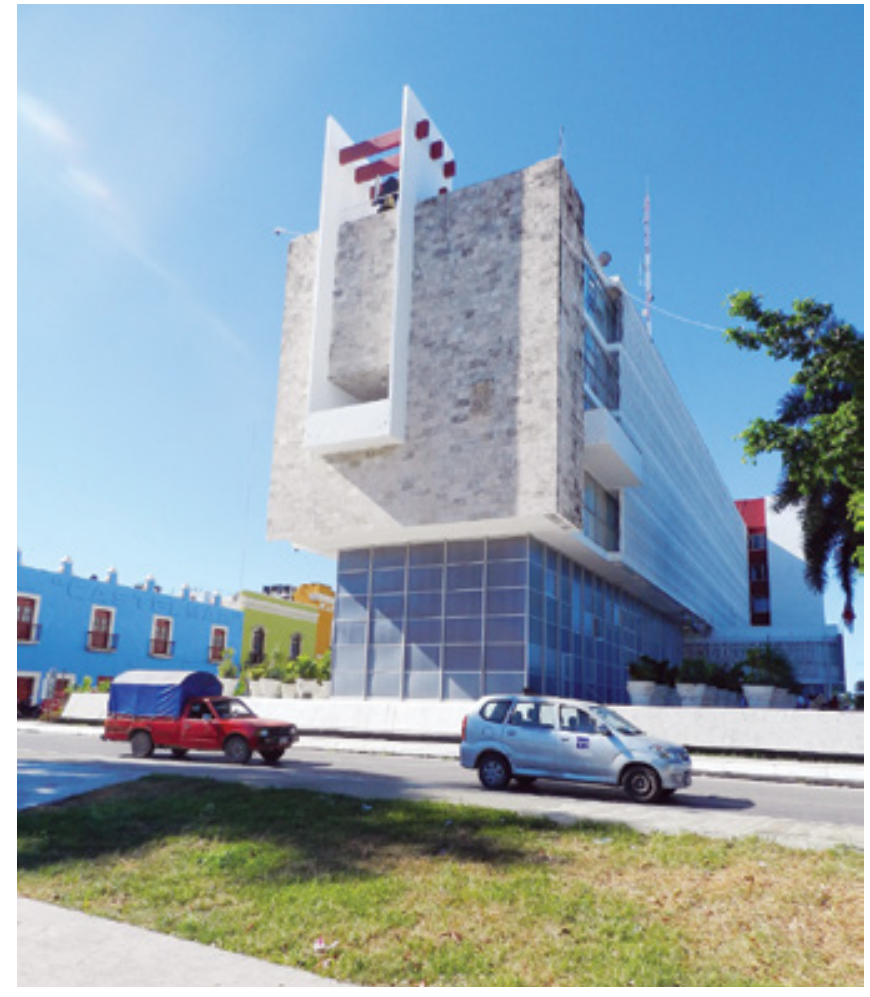

FIGURA 6. Edificio de los Poderes, ciudad de Campeche, Campeche, México: se puede observar que ya no conservan las escaleras con dirección a la plaza y que la planta baja ha sido cubierta (Fotografía: Raúl González Medina 2013). 
evaluación de los inmuebles y la tendencia conservacionista en algunas de ellas, propiciadas por la idea de priorizar la imagen colonial de Campeche, ponen en peligro las construcciones edificadas durante el periodo 19611962. La falta de valoración de las obras modernas ha causado, asimismo, que la mayoría estén en muy malas condiciones, lo que pone en riesgo su permanencia.

La problemática planteada se observa claramente en las alteraciones que ha sufrido el Centro Cívico Héctor Pérez Martínez en sus pavimentos y su estructura espacial; actualmente ya es difícil distinguir la nave original del mercado Pedro Sainz de Baranda debido a las transformaciones de que ha sido objeto y a que ha propasado su capacidad, ocasionando el adosamiento de puestos improvisados en sus andadores; la fuente de los Pescadores, en el barrio de San Francisco que aún conserva habitantes dedicados a la pesca, fue demolida para ser sustituida por una réplica que se encuentra a unos cuantos metros del sitio original. También es notable el deterioro y las modificaciones de otras edificaciones modernas, como el Edificio de los Poderes con la construcción de un elevador y remodelaciones en su interior, así como la Cámara de Diputados con ampliaciones en la parte inferior, y la Plaza de la República, la cual ha perdido su conceptualización original de la serpiente y el águila para dar paso al muelle que resalta la puerta de mar del Campeche amurallado, perdido, así, por completo, su vínculo - ya mermado por la eliminación de las escaleras que los unía conceptualmente-con el Edificio de los Poderes.

Si bien desde 2003 se fundó el capítulo mexicano de Documentación y Conservación del Movimiento Moderno en arquitectura (DOCOMOMO) que tiene como fin la documentación, el registro y la conservación del patrimonio arquitectónico del siglo pasado, en el estado de Campeche no ha tenido fuerte impacto en la preservación de este patrimonio, como tampoco en el fomento de una cultura de conservación y la difusión de los valores de este periodo no sólo desde el punto de vista arquitectónico sino también cultural, social y económico.

Para ejemplificar mejor esta realidad, citamos las conclusiones del XV Simposio Interamericano de Conservación del Patrimonio Monumental emitidas en octubre de 1994:

Que la escasez de recursos destinados por sociedades y gobiernos al estudio y preservación de este patrimonio hace ineficaces las acciones que se inscriben en un marco más amplio que el de las intervenciones puntuales; debido a fallas en las leyes y reglamentos o en su aplicación, proliferan las inserciones de construcciones modernas y discordantes con el contexto histórico, como es el caso del palacio de gobierno y la Cámara de Diputados de la ciudad de Campeche (ICOMOS México 2000:110).

Parte de las recomendaciones de este simposio fue que Campeche se promoviera como patrimonio mundial y, ante esta postura, se subvaloró la obra artística de la ciudad vinculada con el centro histórico. No obstante, en el Tercer Encuentro Nacional de Arquitectura del Siglo Xx, celebrado en Puebla durante los primeros días del mes de junio de 2006, se propuso:

Sobre el caso Campeche, sensibilizar a las autoridades locales, y en particular a la oficina relacionada con el sitio inscrito en la Lista del Patrimonio Mundial, sobre los valores de la arquitectura moderna, en especial de aquel patrimonio relacionado directamente con la zona declarada (ICOMOS México 2007:111).

Esto demuestra que la valoración del patrimonio del siglo XX se ha ido transformando con el tiempo y que si bien en el pasado su aliento vanguardista colaboró con la desaparición de importantes construcciones en el área de la plaza principal y con la pérdida de parte del escenario histórico del Campeche colonial e independiente, ahora son un legado irreemplazable, prueba de la evolución de la ciudad.

Por lo anterior, se considera indispensable la intervención de colectivos profesionales, medios de comunicación y entidades gubernamentales que genere conciencia del compromiso social que representa la conservación, revitalización, catalogación, protección y conocimiento de la producción del movimiento moderno en el país, pues, a pesar de haberse edificado en tiempos cercanos, es parte de nuestro patrimonio.

La realidad de hoy en Campeche no ha cambiado mucho: persiste la preponderancia hacia el Campeche colonial, la ciudad y el puerto amurallado, lo que está originando más pérdidas del patrimonio moderno, pérdidas que también son parte de la memoria histórica de la ciudad. En un afán de recrear escenográficamente la plaza principal de la ciudad, con el edificio del poder político, se reconstruyó el antiguo palacio de gobierno, pero sólo en su sección central en la primera etapa, continuando la construcción de las otras secciones en la actualidad. El uso de este edificio semirreconstruido es de biblioteca, pero, para darle el estatus de edificio gubernamental aunque sea por una sola noche al año, se trasladaron la campana y el acto del grito de Independencia, ceremonia que se celebraba, unida a la tradición de la Feria de San Román, en el Edificio de los Poderes. Al habérsele negado esta función, la Plaza de la República (Figura 7), contenedora del pueblo que asistía al acto, pasó a ser ya un espacio abierto que funcionó un tiempo como estacionamiento y que ahora, con su nuevo diseño de muelle, le impide mantener las funciones que propiciaba su diseño original, es decir, como la plaza del Edificio de los Poderes.

Nuevamente, este espíritu histórico del Campeche amurallado ha llevado al gobierno a destruir y desvincular por completo a la Plaza de la República del Edificio de los Poderes. Un nuevo diseño para escenificar el paisaje 


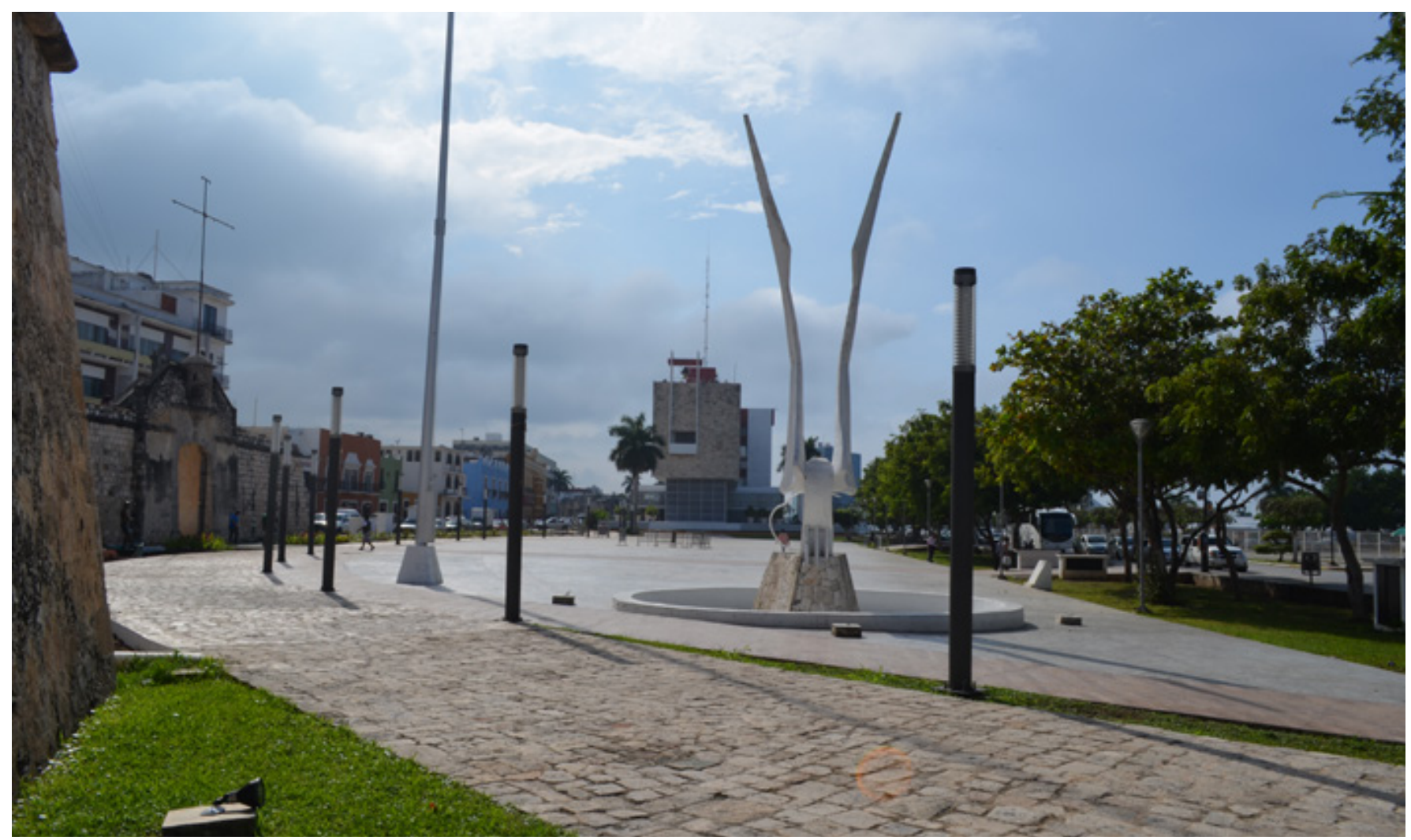

FIGURA 7. Vista de la Plaza de la República desde el Baluarte de la Soledad, ciudad de Campeche, Campeche, México, con el nuevo diseño (Fotografía: Aurelio Sánchez 2013).

marino junto a la puerta de mar ha cambiado el concepto y la forma de la plaza; se han construido desniveles para albergar cuerpos de agua y construir un simulacro de muelle frente a dicha puerta. ${ }^{4}$ El águila, que era representativa de la plaza, quedó aislada de su contexto y preservada como una pieza de museo. Así, terminamos este trabajo preguntándonos: ¿será posible que sea parte de este concepto de ciudad museo? Lo cierto es que este diseño, que empieza en el otro extremo del baluarte de la Soledad, no fue del todo funcional al cambiar el agua por área verde, amén de que queda inconcluso por razón de que el proyecto de continuar la reconstrucción de la muralla traerá quizás nuevas ideas escenográficas.

Pareciera ser que la idea de conservación del patrimonio moderno en Campeche es la demolición de monumentos y su reconstrucción a pocos metros de su sitio original, en lugar de buscar soluciones urbanísticas y diseños morfológicos que privilegien la conservación del patrimonio edificado. Como ejemplo, baste señalar la fuente de los Pescadores (Figura 8), ubicada, como se ha dicho, en el barrio de San Francisco; la fuente del Progreso, emplazada junto al baluarte de San Carlos sobre la lí-

\footnotetext{
${ }^{4}$ El reciente proyecto de recuperación y puesta en valor de la imagen urbana de la ciudad histórica de San Francisco de Campeche propone reconstruir el lienzo de muralla que falta, para dar en la plaza la imagen general del mar golpeando la muralla.
}

nea de la construcción del lienzo de muralla, y la escuela primaria Justo Sierra, en el extremo oriente extramuros del recinto amurallado, los cuales se demolieron y reconstruyeron a escasa distancia de su primer emplazamiento, ${ }^{5}$ con la pérdida de su valor de autenticidad, en decisiones que privilegiaron el diseño urbano de las vialidades ante la conservación del patrimonio moderno.

Campeche ha tenido características únicas en su patrimonio edificado, como lo fueron los portales mixtilíneos en su Plaza de Armas, destruidos, junto con las fachadas de los edificios que complementaban el frente de la cuadra, para dar paso, quizá, a la idea de arquitectura colonial de los portales de arco de medio punto, ya con la falta de los portales del antiguo palacio de gobierno. En su concepción y temporalidad, se pretendía que la construcción de arquitectura considerada hoy patrimonio moderno se integrara al patrimonio histórico. No obstante que ocupó espacios colindantes y cercanos al centro histórico de la ciudad, su diseño procuró mantener la visual al mar, aunque estilísticamente no se integró al patrimonio ya edificado. Como todo proceso de apropiación y valoración, fue rechazado por tendencias conservadoras. Hoy en día este proceso de apropiación y valorización del patrimonio moderno no se ve en Campeche. Se ha

${ }^{5}$ A excepción de la fuente del Progreso, que se reconstruirá en el barrio de Guadalupe. 


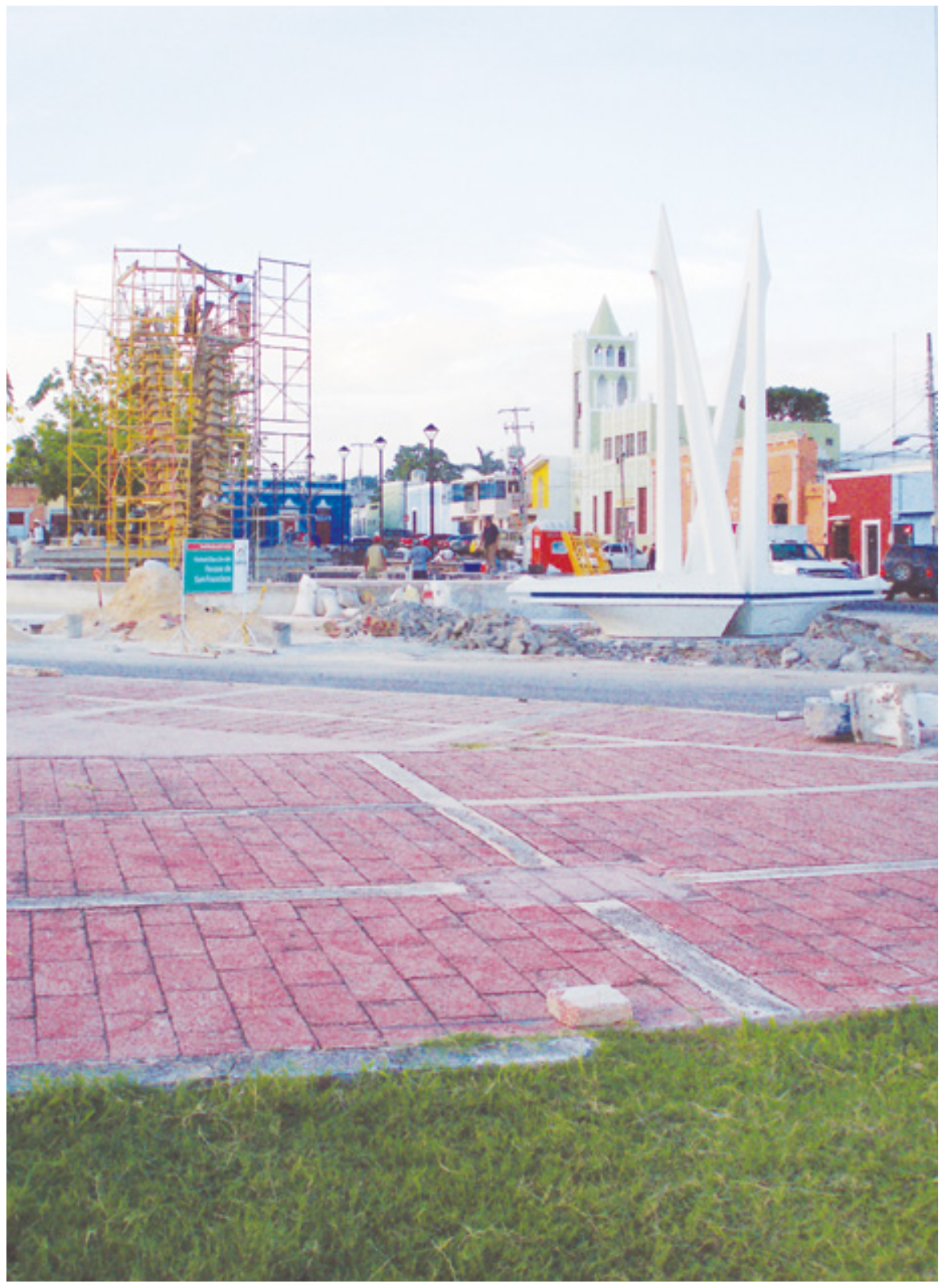

FIGURA 8. Reconstrucción de la fuente de los Pescadores, ciudad de Campeche, Campeche, México, a unos metros de la original, misma que fue demolida posteriormente (Fotografía: Raúl González 2013).

perdido parte del patrimonio moderno, se ha desfigurado su morfología, y si no se propicia el diálogo entre especialistas de las diferentes categorías del patrimonio, seguirá preponderando uno sobre otro, con la consecuente destrucción del más débil.

\section{Referencias}

Calduch Cervera, Juan

2009 "El declive de la arquitectura moderna: deterioro, obsolescencia, ruina", Palapa IV (11): 29-43.

\section{CEUM}

1972 "Ley Federal sobre Monumentos y Zonas Arqueológicos, Artísticos e Históricos", Diario oficial de la Federación.

Conti, Alfredo

2003 El patrimonio moderno en la Lista del Patrimonio Mundial, México, Conaculta/INAH.

Díaz-Berrio Fernández, Salvador 1999 "Teoría de la restauración: avance o retroceso", XVIII Simposio Internacional ICOMOS, Guadalajara, Jalisco, México.

GEC

1962 Inmuebles del gobierno del estado, Campeche, GEC.

1963 Programa de Desarrollo Urbano de Campeche, Campeche, GEC.

ICOMOS

2007 "Conclusiones", Tercer Encuentro Nacional de Arquitectura del Siglo XX, Puebla, ICOMOS.

INAH

2000 Conservación del patrimonio monumental. Veinte años de experiencias, México, INAH.

Lanz, Manuel A.

1905 Compendio de historia de Campeche, Campeche, El Fénix.

Leal Sosa, Jacqueline 2003 La plaza como eje rector de la vida en Campeche, Campeche, Conaculta/INAH.

Muñoz Viñas, Salvador 2003 Teoría contemporánea de la restauración, Madrid, Síntesis.

Pérez Sosa, Artemio

2000 Historia de Campeche: 460 años después de su fundación, 1540-2000, México, Prado.

Sierra, Carlos Justo

1998 Breve historia de Campeche, México, El Colegio de México/FCE.

Silva, José de Santiago

2001 José Chávez Morado: vida, obra y circunstancias, México, Instituto Estatal de la Cultura de Guanajuato (IECG).

UNESCO

1964 "Carta Internacional sobre la Conservación y la Restauración de Monumentos y Sitios", II Congreso Internacional de Arquitectos y Técnicos de Monumentos Históricos, Venecia, Italia. 


\section{Raúl González Medina}

Arquitecto independiente, México glez_raul@hotmail.com

Licenciado en arquitectura (Universidad Marista de Campeche [UMarista-Campeche], México) con la tesis "El patrimonio moderno en la ciudad de Campeche (1961-1962)". Ha participado en proyectos de investigación vinculados con el patrimonio moderno. Actualmente labora como diseñador arquitectónico e investigador independiente.

\section{Aurelio Sánchez Suárez}

Centro de Investigaciones Regionales (CIR),

Universidad Autónoma de Yucatán (UAdY), México

aurelio_suarez@yahoo.com.mx

Doctor en arquitectura (Universidad Nacional Autónoma de México [UNAM], México) y posdoctorado en humanidades (Colegio de Ciencias y Humanidades [CCH], UNAM, México). Investigador titular de la unidad de ciencias sociales del Centro de Investigaciones Regionales (CIR), Universidad Autónoma de Yucatán (UAdY), México. Imparte cátedra y dirige tesis en los posgrados de la Escuela Nacional de Conservación, Restauración y Museografía (ENCRYM), Instituto Nacional de Antropología e Historia (INAH); en la Escuela Superior de Ingeniería y Arquitectura (ESIA), Instituto Politécnico Nacional (IPN), unidad Tecamachalco, y en el posgrado en patrimonio y desarrollo sustentable de la Universidad Autónoma de Campeche (UAC), todos en México. Ha sido titular de proyectos de investigación y publicaciones en revistas nacionales e internacionales sobre cultura maya, arquitectura vernácula, conservación del patrimonio cultural, espacios abiertos y transculturación urbana. Miembro del Sistema Nacional de Investigadores (SNI), Consejo Nacional de Ciencia y Tecnología (Conacyt) y Consejo Internacional de Monumentos y Sitios (ICOMOS), México. 